\title{
Reusabilidad y reutilización de objetos didácticos: Mitos, realidades y posibilidades
}

\author{
Reusability and reuse of learning objects: Myths, realities and possibilities
}

\author{
Miguel-Angel Sicilia \\ Universidad de Alcalá, Alcalá de Henares, España \\ msicilia@uah.es
}

\begin{abstract}
El concepto de objeto didáctico u objeto de aprendizaje (learning object) se ha convertido en el centro de un nuevo paradigma de diseño de actividades de aprendizaje on-line, que hace énfasis en la reutilización de contenidos y actividades, mediante el uso de metadatos en formatos conocidos. En este artículo se revisa la definición del concepto, y se trata de ofrecer un marco conceptual para la justificación del nuevo paradigma y para la delimitación de sus propiedades esenciales. Concretamente, los conceptos de reuso y reusabilidad se analizan en sus diferentes aspectos.
\end{abstract}

Palabras clave: Objeto didáctico, reusabilidad.

\begin{abstract}
The concept of learning object has become the centre of a new paradigm for the design of on-line learning activities, which emphasizes content and activity reuse through the use of metadata in wellknown formats. This papers reviews the definition of the concept and aims at offering a conceptual framework to justify the new paradigm, delimiting also its essential properties. Concretely, the concepts of reuse and reusability are analyzed in their diverse aspects
\end{abstract}

Keywords: Learning object, Reusability.

\section{Introducción}

El concepto de objeto didáctico - del inglés learning object (LO) - ha evolucionado en los últimos años como la noción subyacente (tácita o explícita) alrededor de la cual se estructura un conjunto de tecnologías y estándares que se presupone conducen a una "industria del aprendizaje" más eficientey evolucionada (Martinez, 2001), o si se prefiere, a un nuevo panorama en la educación basada o apoyada en la Web. Desde el punto de vista teórico, de este simple enunciado se derivan al menos dos cuestiones abiertas de importancia, que pueden formularse de la siguiente forma: (a) ¿cuáles son las ventajas del paradigma de los LO? (es decir, por qué debemos adoptarlo y preocuparnos por él) y (b) ¿cuál debe ser la definición de LO y sus características esenciales?, para que esas ventajas se hagan realidad. Dicho de otro modo, es preciso aclarar los presupuestos o hipótesis del paradigma de los LO, dado que, como en 
cualquier otra disciplina, las racionalidades evaluativa y práctica (Bunge, 1985) ${ }^{1}$ requieren establecer: (i) si las metas deseadas son alcanzables (esto es, si son factibles), (ii) si merece la pena alcanzarlas (esto es, si son deseables) y (iii) cuáles son los medios que pueden ayudar eficazmente a alcanzarlas. Por otro lado, el estado de relativa madurez de la disciplina, junto a la aparición de los primeros resultados significativos (y con ellos, de las primeras críticas) hace posible y deseable una reflexión sobre qué se pretende con el paradigma de los LO, y cuáles son los pasos que deberíamos dar a partir del momento actual para conseguirlos ${ }^{2}$. Como en muchas otras áreas de estudio, los objetivos originales de un nuevo concepto a menudo se difuminan con el tiempo, y se corre el riesgo de adoptar los LO o sus presupuestos como "artículo de fe", sin otro criterio que seguir la aproximación ortodoxa o "de moda".

Antes de continuar, es necesario al menos identificar cuál o cuáles son las características de los LO que los hacen diferentes o "mejores" que otras aproximaciones al aprendizaje en la Web que nos los toman en cuenta. Es un hecho que no existe una definición comúnmente aceptada del término LO, y más bien el problema de la definición es una controversia no resuelta (algunos dirían irresoluble). No obstante, si examinamos las definiciones existentes, encontraremos que todas ellas tienen como elemento común el hacer algún tipo de referencia, tácita o explícita, a la reutilización de los mismos. Esta reutilización repetida es de hecho la fuente de los potenciales beneficios del concepto. Desde un punto de vista económico, es fácil construir modelos de costebeneficio para justificar el diseño de LO, análogos en cierta medida a los modelos que han

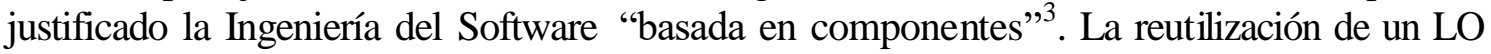
- dentro de una misma organización o en diversas de ellas - es un hecho empírico observable y sujeto a ser registrado dentro de un esquema económico. Cuestión bien diferente es el de la reusabilidad de un LO, que entra en la categoría de atributos "intrínsecos" de artefactos, y por tanto, que puede utilizarse como medida de calidad a priori. En el caso de la reusabilidad - como ocurre en la disciplina de la Ingeniería del Software - no existen medidas precisas, sino sólo indicadores, que podrán o no ser confirmados por tasas de reutilización elevadas a posteriori, sin que desdigan en nada la potencial reusabilidad. En consecuencia, pensar y trabajar en o sobre LO implica de algún modo pensar en reutilización, o dicho de otro modo, el criterio de demarcación de diseños en el paradigma de LO es la orientación decidida a la reutilización, lo cual determina en parte los criterios de buen diseño y descripción - técnico y pedagógico - de los contenidos Web. Sorprendentemente, existen muy pocos estudios actuales sobre reutilización práctica de L, y aún menos sobre indicadores de reusabilidad, aunque sí hay un buen número de trabajos que proporcionan directrices más o menos vagas para el diseño de LO.

En el resto de este artículo, pretendemos responder a las preguntas (a) y (b) anteriores desde h perspectiva de la reutilización, así como plantear en consecuencia respuestas para las subsiguientes preguntas (i) a (iii), de modo que, si así lo conseguimos, podamos continuar trabajando en la dirección de los LO desde una perspectiva clara de hacia dónde

\footnotetext{
${ }^{1}$ La racionalidad evaluativa implica "bregar por metas que, además de ser alcanzables, valga la pena alcanzar" y la racionalidad práctica implica "adoptar medios que puedan ayudar a alcanzar las metas propuestas".

${ }^{2}$ De hecho, la red de excelencia Prolean (http://www.prolearn-project.org) tiene entre sus objetivos el establecer este tipo de "mapa de carreteras".

${ }^{3}$ No olvidemos que cualquier learning object podría considerarse en último término como una pieza de software, aunque de un tipo muy específico.
}

Reusabilidad y reutilización de objetos didácticos: Mitos, realidades y posibilidades. Miguel-Angel Sicilia 
debe orientarse la racionalidad tecnológica y los medios materiales disponibles. Nuestra discusión ha de incluir, por supuesto, hasta qué punto los actuales estándares y especificaciones de LO alcanzan las metas deseadas, y cuánta parte de los objetivos que en ocasiones se enuncian como ventajas de la aproximación de los LO son (de manera contingente o definitiva) simples mitos o ideas vagas diseñadas con objetivos espurios o ingenuos.

\section{Reusabilidad de los Objetos Didácticos}

La característica esencial de un objeto didáctico es la "predisposición al reuso". De un modo u otro, este aspecto aparece en las definiciones más populares del término. Polsani (2003) incluye la reutilización en su definición de learning objects como "una unidad independiente y auto contenida de contenido de aprendizaje que esta predispuesta a ser reutilizada en múltiples contextos de aprendizaje", y Wiley (2001) también menciona el término en su definición de learning object "cualquier recurso digital que puede ser reutilizado para proporcionar aprendizaje". Por otro lado, desde una perspectiva económica, resulta claro el que el uso repetido es la fuente de valor y de economía de escala para el caso de los proveedores de contenidos. Una consideración central a nuestro análisis es que la reusabilidad depende de los metadatos tanto como del contenido, en el escenario futuro en el cual el acceso a los LO se haga por herramientas software. Los metadatos son (o deberían ser) descripciones orientadas fundamentalmente a las máquinas, y no a la lectura humana. Pensar en la reutilización a gran escala sin la mediación de software especializado es perder la perspectiva del fenómeno que pretendemos caracterizar. Esto nos lleva a exponer el primer mito ampliamente extendido.

Mito \#1: Proporcionar un registro de metadatos compatible con LOM (IEEE, 2002) o un empaquetado compatible SCORM ${ }^{4}$ hace a LO reutilizable.

Desgraciadamente, el crear metadatos LOM - aunque evidentemente útil - no garantiza per se la reusabilidad, debido a que esos metadatos pueden ser erróneos o incompletos, y aún más, pueden ser aptos para la lectura humana pero no para el procesamiento automatizado, con lo cual tenemos el mismo problema de los buscadores en la Web. Lo mismo puede decirse de SCORM, excepto para el significado restringido que comentamos a continuación.

Realidad \#1: SCORM permite el intercambio de contenidos entre plataformas de elearning, lo cual es un tipo de reutilización de carácter técnico (es decir, de intercambio de archivos).

Esta sí es una realidad incontestable. SCORM proporciona reusabilidad en el sentido de posibilidad de intercambio. Por supuesto, un contenido SCORM puede ser muy poco reutilizable, por ejemplo, por ser de granularidad grande, o simplemente por ser muy específico de una situación determinada.

\footnotetext{
${ }^{4}$ http://www.adlnet.org 
Posibilidad \#1: Basándonos en LOM y en SCORM, se pueden conseguir altos grados de reusabilidad, siempre que se tenga cuidado de que los metadatos tengan formatos adecuados para su procesamiento automatizado.

Un ejemplo de esfuerzo en la dirección de la posibilidad \#1 es el del "diseño por contrato" de LO (Sicilia \& Sánchez, 2003), que hace énfasis en proporcionar una semántica clara de los metadatos para que las herramientas software puedan seleccionarlos y combinarlos, verdaderas tareas de reutilización que hasta la fecha realizaban pacientemente tutores y profesores "cortando y pegando" o combinando fuentes bibliográficas diversas. Entonces podemos considerar ya varios aspectos de la reusabilidad:

1. Un aspecto técnico de formato que implica que los materiales estén formateados de acuerdo a ciertas reglas y convenciones. Esto lo consiguen en gran medida los estándares actuales.

2. Un aspecto técnico de interpretación que implica que los metadatos utilizados tengan una orientación a habilitar ciertas funcionalidades automatizadas conocidas, de manera precisa. LOM no es suficiente en esta área, pero puede extenderse con técnicas y prácticas para hacerlo.

3. Un aspecto de diseño instruccional, de manera que el diseño de los contenidos y su granularidad esté orientado a la reutilización, pensando en posibles entornos de uso futuros.

Para el tercer aspecto no existen fórmulas mágicas, pero sí se ha propuesto al menos un esbozo de caracterización del concepto (Sicilia \& García, 2003) que relaciona la usabilidad en diferentes contextos educativos del LO con la reusabilidad total.

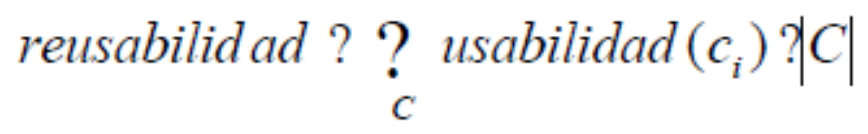

En el caso de un objeto con un solo contexto de uso determinado, la reusabilidad tendría la unidad como valor máximo. Sin embargo, un objeto con seis contextos de uso establecidos por sus metadatos podría tener un valor de reusabilidad que oscile entre $6 \mathrm{y}$ -6 , indicando los valores negativos que hay una mala adecuación de la usabilidad para un contexto educativo determinado. El valor de la expresión descrita es que nos indica que la reusabilidad aumenta, como la intuición nos indica, con el incremento de los posibles contextos de uso para el objeto, pero con la condición de que los contenidos y estructura del mismo sean apropiados para todos ellos. Así, el diseño del objeto y de sus metadatos tendrá que tratar de definir lo más precisamente posible los contextos posibles de uso, y la adecuación a cada uno de ellos será objeto fundamental de la evaluación.

Por supuesto que la caracterización de la reusabilidad descrita es a posteriori, en el sentido de que requiere la evaluación del LO en diferentes entornos. Sería deseable complementarla con otras medidas o indicadores a priori, para lo cual podría explorarse el concepto de reusabilidad en Ingeniería del Software.

Por otro lado, hay que tener en cuenta de que el paradigma de los LO sólo resultará útil si se da en la práctica una adopción generalizada. De aquí viene el segundo de los mitos de uso común que analizamos.

Reusabilidad y reutilización de objetos didácticos: Mitos, realidades y posibilidades. 
Mito \#2: Hay una enorme cantidad de contenidos educativos reutilizablesen repositorios Web.

No es cierto que haya tal cantidad de recursos ya disponibles, y esto no es sorprendente, ya que la creación de metadatos es una actividad costosa. Lógicamente esta afirmación puede ser discutida, ya que el concepto de "gran cantidad" puede interpretarse de distintos modos. En cualquier caso, podemos decir que aunque haya muchos recursos, hay pocos con los metadatos adecuados. A este respecto, el estudio de Sicilia et al. (2004) y el de Najjar et al. (2003) son de los pocos informes que muestran algo de evidencia sobre la realidad de los metadatos en repositorios de uso común. No obstante, existen ya notables casos de repositorios de LO como MERLOT o CAREO con una considerable cantidad y variedad de contenidos, por lo cual podemos afirmar lo siguiente.

Realidad \#2: Existen ejemplos de repositorios de LO con buenas y amplias bases de datos de contenidos, que evolucionan de manera constante.

De hecho, una cierta mejora en las interfaces y técnicas para recoger los metadatos en esos repositorios (el aspecto técnico de interpretación) podría resultar en una verdadera disponibilidad de LO para las aplicaciones que resultase en una extensión del paradigma y de la tecnología asociada.

Por otro lado, el uso de nuevas tecnologías como las de la Web Semántica (Berners-Lee, 2001) prometen enriquecer los metadatos para un aprovechamiento que realmente signifique un cambio relevante el diseño de programas de aprendizaje, con herramientas de búsqueda mejoradas.

Posibilidad \#2: Con tecnología disponible, puede construirse una infraestructura que facilite de manera significativa la reutilización de LO.

De todo lo anterior se desprende un mito adicional.

Mito \#3: El paradigma de los LO ha demostrado su utilidad en la construcción de una nueva industria y arte de las actividades de aprendizaje on-line.

Dicha afirmación es falsa, en el sentido de que la evidencia necesaria para apoyarla aún no se ha recopilado. No existen estudios sistemáticos de reuso que permitan apoyarla.

\section{Puntos de Vista para el Diseño y evaluación de la Reusabilidad de un Objeto Didáctico}

Los motivos que hacen que el paradigma de los LO tenga un valor sobre aproximaciones existentes al diseño de contenidos y actividades educativas son de diferente índole, y pueden resumirse mediante perspectivas o puntos de vista. A continuación describimos brevemente algunos de estos puntos de vista, en un intento de responder a la cuestión (a) que planteamos al principio de este artículo.

Reusabilidad y reutilización de objetos didácticos: Mitos, realidades y posibilidades. 


\section{Punto de vista de la generación de valor}

La noción de valor es el fundamento de la disciplina de los Sistemas de Información (information systems) en el contexto organizativo. El valor admite muchas definiciones, incluyendo elementos de carácter puramente económico y también otros relativos a la capacidad de servicio. En cualquier caso, un modelo de valor simplista dentro de una organización podría expresarse en términos coste-beneficio, siendo el coste el valor de la adquisición o producción de el o los LO necesarios para una determinada necesidad formativa, y el beneficio el incremento en las competencias o conocimiento de los individuos que se benefician de la acción formativa basada en ellos. Hay que tener en cuenta que en el contexto organizativo, las actividades orientadas al aprendizaje forman parte de un ciclo de adaptación como el que se muestra en la siguiente Figura.

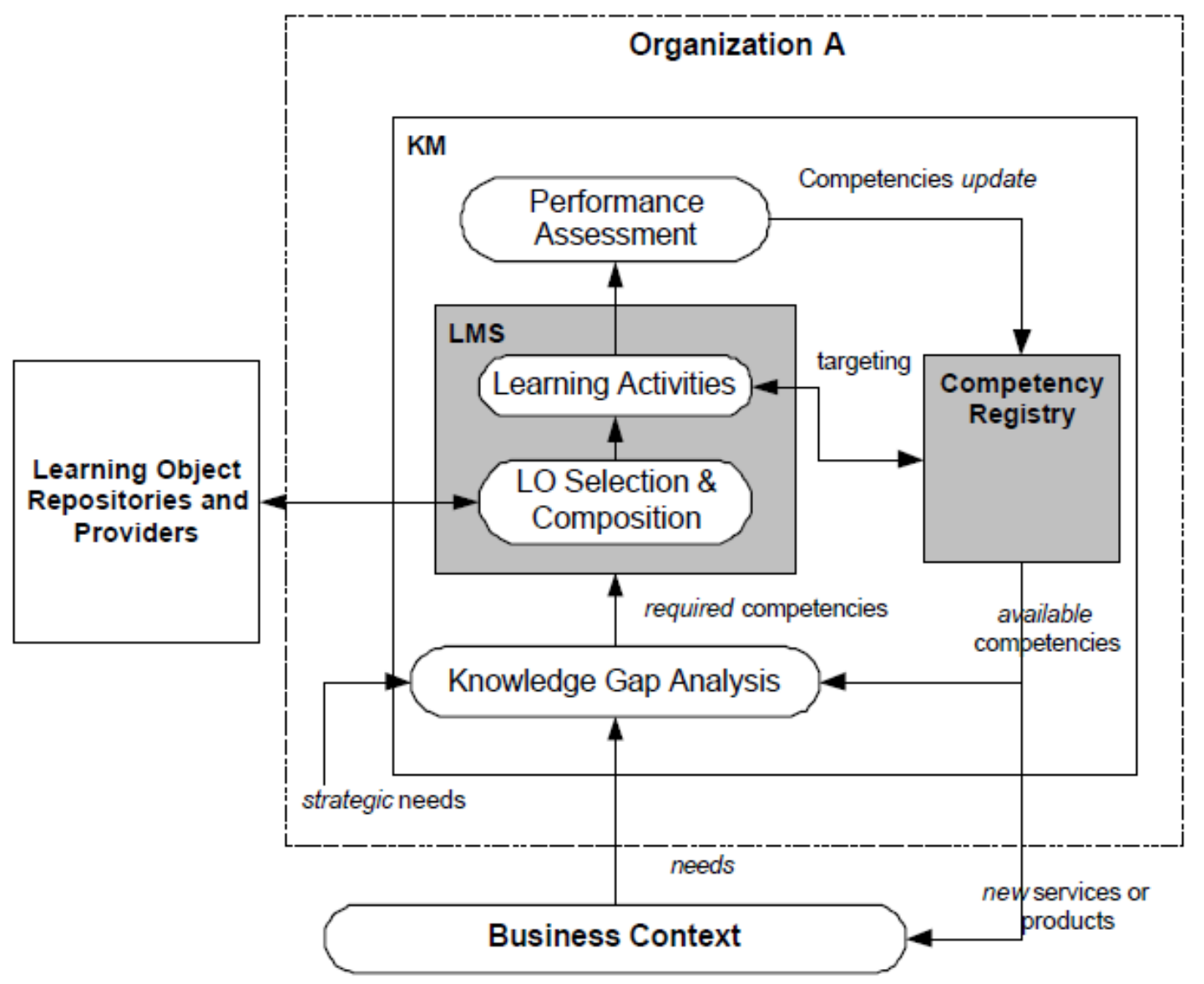

De este modo, el valor se conceptualiza como un incremento final en la capacidad competitiva de la organización. El uso de proveedores externos de LO permite centrarse a la organización en sus capacidades competitivas, usando en modalidad de outsourcing las actividades de aprendizaje. Por otro lado, las economías de escala a la que pueden llegar los fabricantes de LO estandarizados llevará con toda probabilidad a una reducción de los costes globales de producción de contenidos y actividades.

\section{Punto de vista de conformidad técnica}


Desde la perspectiva de la administración de los sistemas de e-learning, los estándares actuales son la base de la interoperabilidad de contenidos y actividades. Poco cabe añadir a esto, ya que su capacidad en esta área está sobradamente probada, y los organismos de especificación continúan una actividad intensa para abarcar más áreas de conformidad.

\section{Punto de vista de adecuación pedagógica}

Desde el punto de vista del tutor o educador que busca LO para una situación concreta, la disponibilidad de herramientas de búsqueda y composición automatizadas, además de ahorrar tiempo en el diseño, amplían las posibilidades de encontrar lo adecuado a las necesidades concretas. A este respecto, cuanto mayor sea el grado de detalle de los metadatos desctriptivos, mayores son las posibilidades efectivas de reuso. Es importante notar que el uso de metadatos estructurados con interpretaciones no ambiguas abre un escenario completamente diferente a la construcción de herramientas de búsqueda, más allá de los buscadores convencionales, con sus conocidas limitaciones en cuanto a precisión y relevancia.

\section{Punto de vista de la cal idad proveniente del uso repetido}

Una característica importante de los contenidos y actividades educativas es que se evalúan y perfeccionan con la práctica, es decir, con su uso repetido. Esto hace que la reutilización, además del beneficio de valor, produce un beneficio derivado en cuanto a calidad incrementada por la evaluación y experiencia repetida. Este efecto es bien conocido en Ingeniería del Software, en el cual los componentes "ampliamente utilizados" tienen una menor probabilidad de esconder defectos ocultos. Por otro lado, sistemas de revisión y comentario organizados como los del repositorio MERLOT añaden una dimensión de meta-información sobre calidad y adecuación que posee un valor intrínseco muy importante.

\section{Conclusiones}

El paradigma de los LO se centra en el énfasis en la reusabilidad de los contenidos y actividades orientadas al aprendizaje. Por tanto, podemos considerar la reusabilidad como la característica esencial de los LO. No obstante, la reusabilidad es un concepto que abarca aspectos de formato, de interpretación y de adecuación pedagógica. Los estándares y especificaciones actuales cubren el primero de los aspectos, pero son necesarias mejoras y nuevos conceptos en los dos segundos aspectos. Por otro lado, hay diferentes puntos de vista - incluyendo perspectivas de valor y calidad -, que justifican el esfuerzo añadido que requiere la producción de los metadatos para los LO.

Fecha de cierre de la redacción del artículo en su primera edición: 12 de febrero de 2005

Aprobación del artículo en su primera edición: 20 de febrero de 2005

Publicación: 15 de julio de 2016

Reusabilidad y reutilización de objetos didácticos: Mitos, realidades y posibilidades. 
Sicilia, M.A. (2016). Reusabilidad y reutilización de objetos didácticos: Mitos, realidades y posibilidades. RED. Revista de Educación a Distancia, 50. Consultado el (dd/mm/aaaa) en http://www.um.es/ead/red/50

\section{Referencias}

Berners-Lee, T., Hendler, J., Lassila, O. (2001). The Semantic Web. Scientific American, 284(5) (pp. 34-43).

Bunge, M. (1985). Racionalidad y realismo. Alianza Universidad.

IEEE Learning Technology Standards Comitee (2002). Learning Object Metadata. IEEE 1484.12.1.

Lytras, M., Pouloudi, A., \& Poulymenakou, A. (2002). Dynamic e-Learning setting through advanced semantics: The value justification of a knowledge management oriented metadata schema. International Journal of e-Learning, 1(4) (pp. 49-61).

Martinez, M. (2001). Successful Learning - Using Learning Orientations to Mass Customize Learning. International Journal of Educational Technology, 2(2). $\begin{array}{llllll}\text { Consultado el } & \text { el } & 12 & \text { de } & \text { febrero } & 2005\end{array}$ http://www.outreach.uiuc.edu/ijet/v2n2/martinez

Najjar, J., Ternier, S. and Duval, E. (2003). The Actual Use of Metadata in ARIADNE: an Empirical Analysis. Proceedings of ARIADNE Conference 2003.

Polsani, P. R. (2003). Use and Abuse of Reusable Learning Objects. Journal of Digital information, 3(4). Consultado el 12 de febrero de 2005 en http://jodi.ecs.soton.ac.uk/Articles/v03/i04/Polsani/

Sicilia, M.A. \& García, E. (2003). On the Concepts of Usability and Reusability of Learning Objects. International Review of Research in Open and Distance Learning 4(2). Consultado el 12 de febrero de 2005 en http://www.irrodl.org/content/v4.2/sicilia-garcia.html.

Sicilia, M.A. and Sánchez-Alonso, S. (2003). On the concept of learning object "Design by Contract". WSEAS Transactions on Systems, 2(3) (pp. 612-617).

Sicilia, M.A., García, E., Pagés, C., Martínez, J.J. and Gutiérrez, J.M. (2005). Complete Metadata Records in Learning Object Repositories: Some Evidence and Requirements. International Journal of Learning Technology (to appear)

Wiley, D. A. (2001). The Instructional Use of Learning Objects. Association for Educational Communications and Technology, Bloomington. 